\title{
Endodontic Retreatment Using Reciprocal File System in Underfilled Root Canal: A Case Report
}

\author{
Ramadhani Putri Salicha and Kun Ismiyatin \\ Department of Conservative Dentistry, Faculty of Dental Medicine, Universitas Airlangga, Surabaya, Indonesia
}

\begin{abstract}
Underfills in endodontic treatment increases failure rates by $14 \%$. A 23 -year-old female patient came with chief complaint of slight pain on \#35. The periapical radiograph showed underfilled root canal and periodontal ligament space widening. The root canal filling was removed with reciprocal file system. Follow-up visit showed no recurrence pain and the tooth function was fully restored using a fiber-reinforced post and an all-ceramic crown.
\end{abstract}

Key Words: reciprocal file system, retreatment, underfill

\section{INTRODUCTION}

The most common causes of endodontic failure include overfill, underfill, ledge formation, perforation, and instrument fracture. Out of 100 patients, underfill were found in $46.9 \%$ of the root canals and for every $1 \mathrm{~mm}$ short of working length in teeth with apical periodontitis, the endodontic failure rates increased by $14 \% .{ }^{1}$ Radiographic evaluation is a common method for assessing the technical quality of RCT (radiochemotherapy). This assessment process is important because the quality of root canal obturation greatly affects the prognosis of therapy. ${ }^{2}$ Poor techniques such as inadequate length determination, filling technique, and excessive curvature in canal morphology may play a role in the incidence of underfill. There are several techniques for removing gutta-percha from previously treated root canals, including hand files, burs and devices, which are generally preceded by softening of the gutta-percha filling with the aid of solvents or heat. However, all techniques leave residual debris in the canal even after re-instrumentation. Therefore, a new concept always evolve and introduced, one of them is accomplished using a specifically designed nickel-titanium engine-driven instrument that employs a reciprocating motion in a single file system. ${ }^{3}$ Reciprocating file system is a single-use file with oscillatory movement and creates greater contact area between the instrument and gutta-percha. The benefits of this file system are shorter working time and efficacy of gutta removal. ${ }^{4,5}$

Complete removal of previous root filling materials is

Paper presented at the Joint Scientific Meeting in Special Care Dentistry, July 5, 2019, Amerta Room, 4th Floor, main campus of Universitas Airlangga, Surabaya, Indonesia.

Corresponding author: Kun Ismiyatin

Department of Conservative Dentistry

Faculty of Dental Medicine

Universitas Airlangga

JI. Mayjen. Prof. Dr. Moestopo No. 47 Surabaya 60132 - Indonesia

Email: kun-is@fkg.unair.ac.id essential for the success of retreatment. Various techniques and materials, including hand files, motor instruments such as rotary and reciprocal file systems, and also chemical agents, have been suggested for effective removal of root canal filling materials. The use of reciprocal file system has been reported to be a more rapid technique for the removal of root canal filling materials compared to the use of another 
instrument..$^{6-8}$ This case report describes the management and resolution of the pain in underfilled root canal after endodontic retreatment on a lower second premolar using reciprocal file system.

\section{CASE REPORT}

A 23-year-old female patient was referred to the Dental Hospital Universitas Airlangga (Conservative Dentistry Department), with the chief complaint of a persistent pain and loss of restoration on second mandibular premolar even after her previous endodontic treatment was done (Figure 1A). The periapical radiograph showed underfilled root canal $3 \mathrm{~mm}$ short from the working length and small periapical radiolucency (Figure 1B). Patient gave a history of root canal treatment in the second mandibular premolar one month before. She stated that her tooth was restored with a temporary restorative materials that was pink coloured, so she demanded to change the restoration with a permanent material. It was diagnosed and previously treated with symptomatic apical periodontitis.

The recommended treatment plan was to attempt endodontic retreatment. The patient understood and agreed to the procedure and consented to treatment on tooth \#35. The case was completed in several appointments.

The temporary crown was dislodged before the first examination. The periapical radiograph was taken before the procedure began. A rubber dam and clamp was placed for isolation. The root filling material was seen and Gates-Glidden drill size 2 were introduced only at the coronal third of the root canal to create a small reservoir for the solvent to soften the gutta-percha before it was removed completely using primary size 25 reciprocating file system (WaveOne Gold, Dentsply, Maillefer, UK). Another periapical radiograph was taken to make sure the total removal of the root filling material. The canal was negotiated to length, according to the electronic apex locator (ProPex Pixi, Dentsply Sirona, UK). The working length measurement was $20 \mathrm{~mm}$. The canal was then re-instrumented to the working length with a size 35 medium (Wave One Gold, Dentsply, Maillefer, UK) file.
After instrumentation, sequence irrigation was done with 2,5\% sodium hypochlorite and 17\% EDTA solution using EndoActivator System (Dentsply/Maillefer) to activate the irrigant and neutralize with sterilized aquadest. Root canal was dried with paper points and obturated using AH Plus sealer and refilled with a matched-taper guttapercha cone. A periapical radiograph was taken to confirm the result of the obturation. A preliminary impression was made with putty of polyvinyl siloxane elastomer (Aquasil soft putty/regular set, Dentsply, Germany) to prepare the temporary crown for the next visit.

In the second appointment, there was no reported recurrence of pain after the retreatment. By using Peeso Reamers, gutta-percha was removed and fiber-reinforced composite was placed. Then, the core built up was completed with dual-cure composite (LuxaCore $Z$ Dual, DMG, America). Crown preparation and double impressions for definitive restoration were taken (Figure 2). An allceramic crown was chosen for the final restorative material. In the final appointment, the crown placement was made with resin cement (RelyX, 3M, US) and the occlusion was checked (Figure 3).

One month follow-up visit showed that there was no recurrence of pain after the retreatment, no periapical radiolucency on radiograph, and the tooth function was fully restored (Figure 4).

\section{DISCUSSION}

Endodontic failure occurs primarily due to infectious causes and procedural errors, where the technical and clinical biomechanical preparation protocols have not been followed. Bacterial elimination from the root canal holds the key to a successful endodontic treatment. ${ }^{9,10}$ The primary factor to achieve this and to prevent future intrusion of bacteria is a thorough and meticulous technique. When these factors are taken into account, success rate has been shown to be as high as $94 \%$. The proper cleaning and shaping technique, and also the measurement of the working length is important. Studies showed that for every $1 \mathrm{~mm}$ loss of working length, the failure rate increases by $14 \%{ }^{1}$

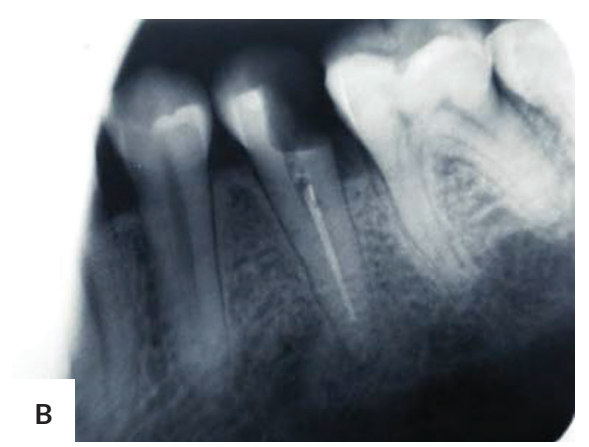

Figure 1. Second mandibular premolar: (A) initial photo, (B) preoperative radiograph showed an underfilled root canal. 

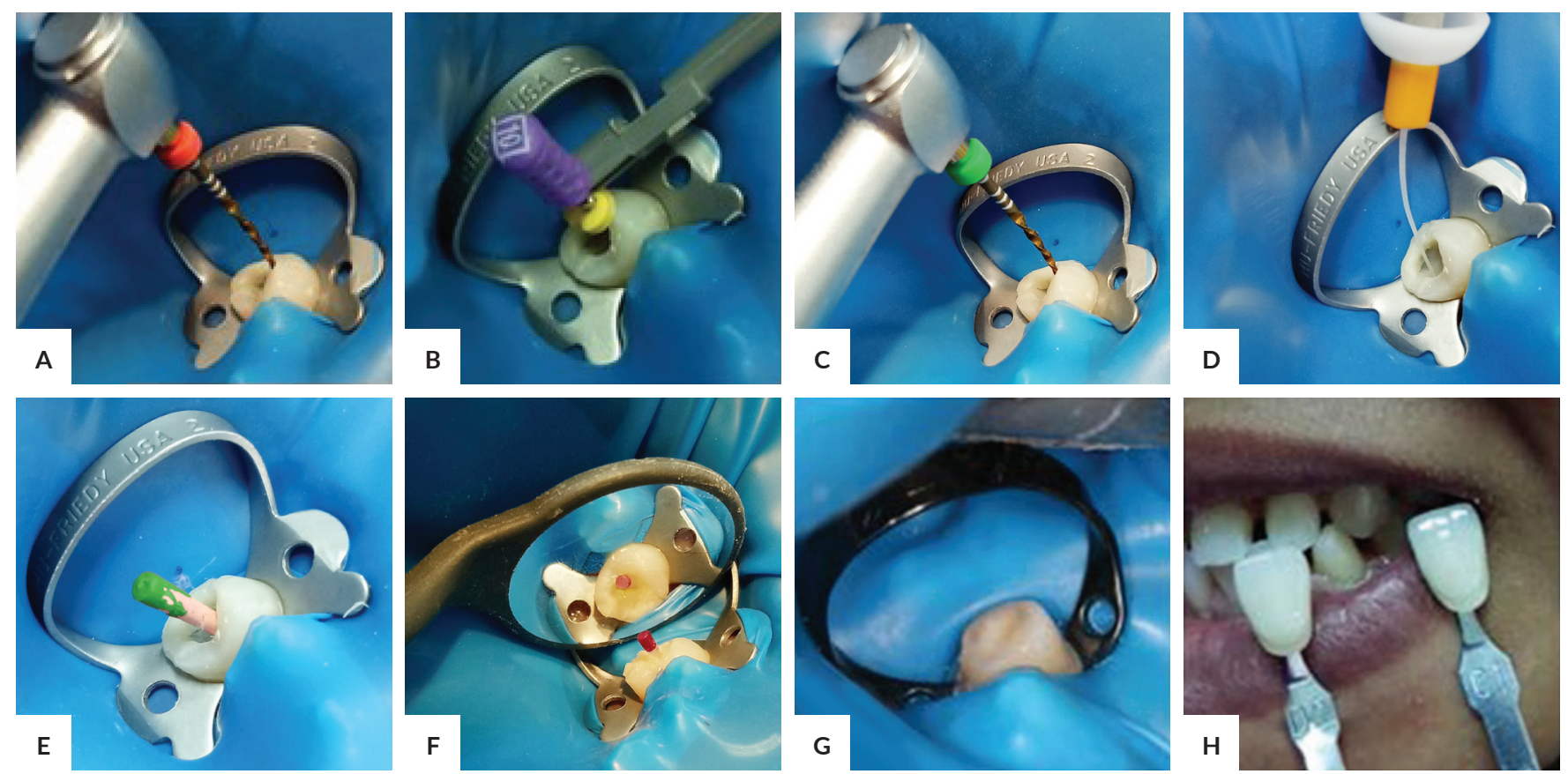

Figure 2. Endodontic retreatment sequence on \#35: (A) Gutta-percha removal with Reciproc file system in 25 primary size (WaveOne Gold, Dentsply/Maillefer, UK), (B) Working length determination, (C) Preparation with Reciproc file system 35 large size (WaveOne Gold, Dentsply/Maillefer, UK), (D) Irrigation with EndoActivator System (Dentsply/Maillefer, UK), (E) Gutta trial, (F) Fiber-reinforced composite placement, (G) Tooth preparation, (H) Shades taking.

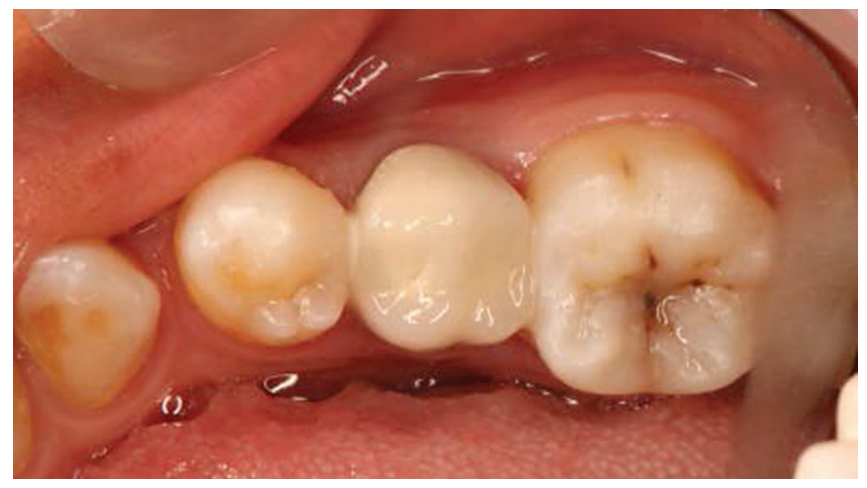

Figure 3. Occlusal view of definitive restoration.

Retreatment is a nonsurgical treatment option for an endodontic failure. Nonsurgical endodontic retreatment is performed to remove material from the root canal space in order to correct deficiencies or repair pathological or iatrogenic defects, followed by cleaning, shaping and threedimensional obturation. Sometimes tooth that has been treated does not heal properly and could become painful or diseased in months or even years after the endodontic treatment. If the tooth fails to heal or develops new problems, it still could be saved through an endodontic retreatmet. An additional procedure may be able to support healing and save the tooth. Retreated tooth can function well for years, even for a lifetime. Advances in technology are constantly changing the way root canal treatment is performed. The endodontist may use a new technique that was not then available during the first procedure. ${ }^{3}$

The removal of old filling materials represents an important step in root canal retreatment, because it enables chemo-mechanical re-instrumentation and re-disinfection of the root canal system. ${ }^{8}$ As for the removal method, the instruments chosen were: Gates-Glidden (GG) drill, ultrasonic devices, hand-use files, motor instruments such as rotary and reciprocal file systems. Some clinicians use solvents to soften the gutta-percha before using rotary or manual instruments, such as xylol/xylene, eucalyptol, methyl chloroform, etc. ${ }^{4,6}$

The reciprocating, alternating or oscillatory movement in Reciproc file system (balanced force), is a system with 


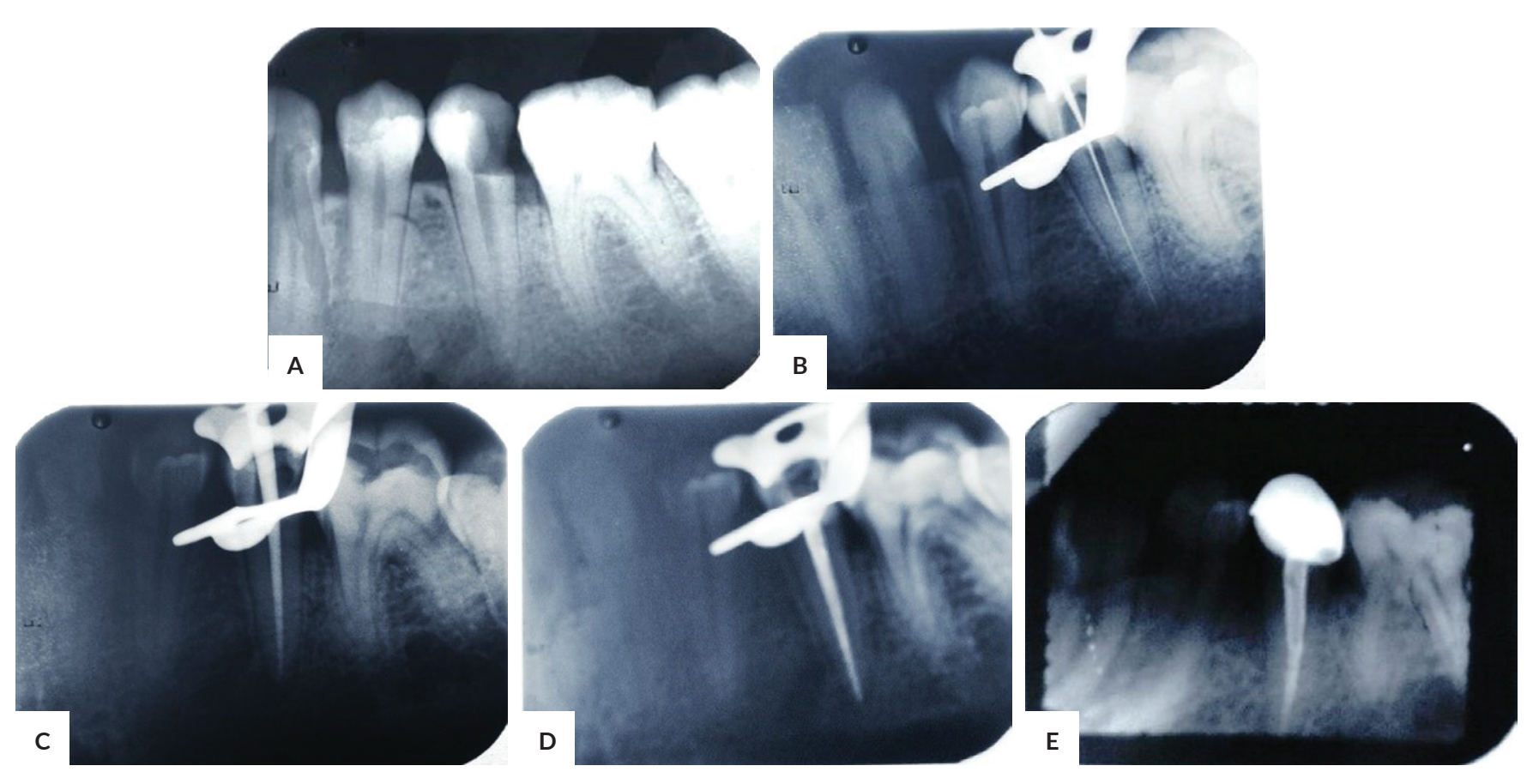

Figure 4. Radiograph pictures on lower second premolar: (A)The removal of old gutta percha filling, (B) Working length determination, (C) Gutta-percha trial, (D) Immediate post-obturation radiograph, (E) 1-month follow-up periapical radiograph showing the fiber post and all ceramic crown.

a single-use file. ${ }^{4,12}$ It is a new concept using a specifically designed nickel-titanium engine-driven instrument that employs a reciprocating motion in a single file system that was developed to aid the retreatment procedure. Reciprocal file system works either as a shaping instrument and also retreatment option because of its specific characteristics and scientific evidence. The system includes nickel-titanium instruments with M-Wire alloy (heated NiTi alloy), which are more resistant to cyclic fatigue, torsional fatigue, greater flexibility, and lower extrusion of debris than conventional alloys. One of the causes of fractures is cyclic fatigue, which is caused by alternate cycles of tension and compression on the alloy. Reciprocal file system works with specific movements. One movement is counter-clock wise (CCW) of 150 degrees, which engages and cuts dentin, and the other is 30 degrees clockwise (CW), which disengages the file from the dentin to avoid taper lock and relieves stress on the file. ${ }^{11,12}$ This motion reduces stress on the instrument and has cutting and release actions. The reciprocal file system is located central in the root canal and creates a greater area of contact between instruments and the guttapercha, thus allowing for better removal of the root filling materials. ${ }^{2}$ Topcu et al, reported that WaveOne (Dentsply/ Maillefer, UK) performed better in canal debridement than Reciproc (VDW). ${ }^{12}$

Reciproc allows the operator to reduce the strength of instrumentation and the number of accidents while doing the treatment, with the advantage of using a single instrument to shape the entire root canal. ${ }^{12}$ It was faster than other systems when removing root canal filling material compared to the other methods. The working time is four times lower than with traditional $\mathrm{NiTi}$ systems.,11,12 On the other hand, systems that utilize equal CW/CCW angles have limitations. These include decreased cutting efficiency, requires more inward pressure, and a limited capacity to auger debris out of a canal. ${ }^{13}$

\section{CONCLUSION}

Retreatment could improve root canal disinfection and debridement before a new homogenous root canal obturation is placed so that the resolution of persistent pain and periapical inflammation could happen. Using the reciprocal file system to remove the previous root filling materials is the most rapid method compared with other retreatment instruments. The result of obturation was the root canal system filled to their full apical length, without any recurrence of pain and periapical radiolucency found on the radiograph, and the tooth function was fully restored using fiber reinforced composite post and an all-ceramic crown.

\section{Statement of Authorship}

All authors participated in data collection and analysis, and approved the final version submitted.

\section{Author Disclosure}

All authors declared no conflict of interest. 


\section{Funding Source}

None.

\section{REFERENCES}

1. Yousuf W, Khan M, Mehdi H. Endodontic Procedural Errors: Frequency, Type of Error, and the Most Frequently Treated Tooth. Int J Dent. 2015;2015: 673914. http://dx.doi.org/10.1155/2015/673914

2. AlRahabi MK. Evaluation of complications of root canal treatment performed by undergraduate dental students. Libyan J Med. 2017 Dec;12(1):1345582.

3. Sahu GK, Consul S, Nandakishore KJ, Shubhashini N, Geeta IB, Idris MD. Rotary Endodontics or Reciprocating Endodontics: Which is New and Which is True ? Journal of Health Sciences \& Research. 2016 Jul-Dec; 7(2):51-7.

4. Galiana MB, Gualdoni GM, Lugo de Langhe C, Montiel NB, Pelaez A. Revisión de desobturación de gutapercha con limas manuales, Xilol y Reciproc. Odontoestomatologia. 2018 Dec; 20(32):12-23.

5. Akbar I. Radiographic study of the problems and failures of endodontic treatment. Int J Health Sci (Qassim). 2015 Apr; 9(2):111-8.

6. Marfisi K, Mercade M, Plotino G, Clavel T, Duran-Sindreu F, Roig M. Efficacy of Reciproc ${ }^{\circledR}$ and Profile ${ }^{\circledR}$ Instruments in the Removal of Gutta-Percha from Straight and Curved Root Canals ex Vivo. J Oral Maxillofac Res. 2015 Jun; 6(3);e1.
7. Keskin C, Sariyilmaz E, Guler DH. Efficacy of novel thermomechanically treated reciprocating systems for gutta-percha removal from root canals obturated with warm vertical compaction. J Dent Res Dent Clin Dent Prospects. 2018 Spring; 12(2);110-5.

8. Kazi FM, Asghar S, Fahim MF. Dissolving Efficacy of Different Endodontic Solvents For Gutta Percha With Varying Time Intervals. J Pak Dent Assoc. 2018;27(3)10-4. DOI: https://doi.org/10.25301/ JPDA.273.110

9. Yuanita T, Ristyawati D, Samadi K. Cytoxicity test of $\mathrm{NaOCl}$ and Mangosteen (Garcinia Mangostin L.) peel extract used as an irrigation solution in human periodontal ligament fibroblast cells (HPdLFc). Dental Journal (Majalah Kedokteran Gigi). 2018;51(3):133-7.

10. Tambayong AJ, Widjiastuti I, Lunardhi CGJ. Differences in photodynamic therapy exposure time and Staphylococcus aureus counts. Dental Journal (Majalah Kedokteran Gigi). 2018;51(2):95-8.

11. van der Vyver PJ, Jonker C. Reciprocating instruments in endodontics : a review of the literature. SADJ. 2014 Oct; 69(9);404-9.

12. Alsilani R, Jadu F, Bogari DF, Jan AM, Alhazzazi TY. Single file reciprocating systems : A systematic review and meta-analysis of the literature : Comparison of reciproc and WaveOne. J Int Soc Prev Community Dent. 2016 Sept-Oct; 6(5):402-9.

13. Ruddle CJ. Single-File Shaping Technique:Achieving a Gold Medal Result. Dent Today. 2016 Jan; 35(1):98 